\title{
Electrospinning Fabrication and In Situ Mechanical Investigation of Individual Graphene Nanoribbon Reinforced Carbon Nanofiber
}

Xinlu Li $^{\mathrm{a} *}$, Yingchao Yang ${ }^{\mathrm{b}}$,Yujie Zhao ${ }^{\mathrm{a}}, J_{\text {un }}$ Lou $^{\mathrm{b} *}$ Xiyu Zhao ${ }^{\mathrm{a}}$, Ronghua Wang, Qinghua Liang ${ }^{\mathrm{c}}$, Zhenghong Huang ${ }^{\mathrm{c}}$

${ }^{a}$ School of Materials Science and Engineering, Chongqing University, Chongqing 400030, China

${ }^{b}$ Department of Materials Science and Nanoengineering, Rice University, Houston, 77005, USA

${ }^{`}$ Key Laboratory of Advanced Materials, School of Materials Science and Engineering, Tsinghua University, Beijing, 100084, China

* Corresponding author:

E-mail address: lixinlu@cqu.edu.cn (Xinlu Li)

¡lou@ rice.edu (Jun Lou) 


\section{Abstract:}

Graphene nanoribbons (GNRs) with opened edges and less structural defects are embedded in polyacrylonitrile (PAN)-based carbon nanofibers (CNFs) by electrospinning followed by stabilization and carbonization. GNRs not only can be used as one-dimensional nanofillers, but also act as nanoplatelet template to promote the formation of graphitic carbon in PAN matrix. X-ray diffraction, Raman spectroscopy and $\mathrm{N}_{2}$ absorption are used to analyze the microstructure of GNR-reinforced CNFs. In situ tensile test using a micromechanical device inside a scanning electron microscope (SEM) is carried out to evaluate the mechanical performance of individual GNR-reinforced CNF. The tensile strength and elastic modulus of CNFs reinforced by 2 wt.\% GNRs with a diameter of $\sim 160 \mathrm{~nm}$ are 3.52 GPa and 70.07 GPa, respectively, which are higher than those of pristine PAN CNFs with similar size, i.e. $2.44 \mathrm{GPa}$ and $28.97 \mathrm{GPa}$. The rough fracture surface for all GNR-reinforced CNFs suggests that the GNRs can dramatically toughen the fibers. A sword-in-sheath failure is observed in 4 wt.\% GNR reinforced CNFs, confirming that GNRs are entirely embedded and well aligned along the fiber axis. This study demonstrates the potential of GNRs as a promising reinforcement to improve the formation of graphitic carbon and mechanical performance of CNFs. 


\section{Introduction}

Carbon nanofibers (CNFs) with high strength to weight ratio are one of the most successful commercialized carbon materials. Owing to their high mechanical strength, unique electrical and good thermal conductivity, CNFs have a wide range of applications, such as carbon based composites, protective textiles, field electron emission sources [1-6]. To date, melt spun[7], gel spun[8, 9], and electrospinning[10-12] are generally used to produce CNFs with several popular polymer precursors including polyacrylonitrile (PAN), pitch, and rayon[13, 14]. Specifically, the CNFs fabricated from PAN have higher a tensile and compressive strength than the fibers produced from rayon, due to the higher melting point and higher carbon yield of PAN (>50\% of the original precursor mass)[14]. Moreover, PAN is much cheaper than pitch[13]. Therefore, PAN, currently, is the main precursor to fabricate high-performance commercial CNFs[15-18].

For PAN-based CNFs, the mechanical properties of electrospinning nanofibers still do not reach the expectation due to the low crystallinity in preparation. Increasing heat treatment temperature in the steps of stabilization and carbonization can improve the degree of graphitization, further resulting in higher mechanical properties of CNFs[11, 12, 19-22], while high temperature undoubtedly will increase the product cost. An alternative effective way to improve the mechanical performance of CNFs is to incorporate nanoscale fillers into the polymer matrix, such as carbon nanotubes (CNTs), graphene oxides and graphene oxide nanoribbons (GONRs), which have 
been used for structural and mechanical reinforcement[2, 5, 8-10, 15, 23-27]. Generally it is believed that the dispersion of nanofillers and interactions between nanofillers and PAN matrix play critical roles in optimizing the reinforcement. For example, several covalent grafting methods including acid oxidation, polymer wrapping, and surfactant modified were developed to functionalize the surface of CNTs in order to improve the dispersion of CNTs in PAN and the interfacial interaction between two phases[7, 9, 24, 25]. Another reinforcement, graphene oxide nanoribbons (GONRs) with a high length-to-width ratio, which can be longitudinally unzipped from CNTs by a chemical oxidation method. GONRs can provide a large interfacial area for $\pi-\pi$ stacking in basal planes with polymer matrix and rich functional groups at the edges, such as carboxylic and hydroxyl groups, which can strongly link GONRs and the polymer matrix together[28, 29]. Therefore, GONRs were used as an effective reinforcement in PAN-based electrospun carbon nanofiber yarns $[8,10,27,30]$. It should be noted that there are a number of structural defects and oxide-functional groups in GONRs, which will largely decrease the mechanical performance of GONRs. In contrast to GONR, graphene nanoribbons (GNRs) with similar opened edges, but less structural defects, which can be easily made from alkali metal intercalated CNTs, have not been investigated as reinforcement in PAN-based CNFs. According to previous reports $[1,3,16]$, the tensile strength of CNFs strongly depends on the diameter. However, the influence of porosity, which acts as structural defects, on the mechanical behaviors of single CNF by has not been studied.

Herein, we choose GNRs as one-dimensional nanofillers reinforcing PAN-based 
CNFs, which are longitudinally split from CNTs by alkali metals. The GNRs not only can be used as the mechanical reinforcement in PAN-based CNFs, but also act as nanoplatelet templates to promote the nucleation of graphitic domains in PAN matrix.

We systematically studied the effect of GNRs on the graphitic crystallinity, specific surface area and porosity at different content. Moreover, we carried out in situ tensile tests in a scanning electron microscopy (SEM) to quantitatively measure the mechanical properties of individual GNR-reinforced CNFs. The effect of GNRs at different weight fraction and porosity of CNFs on the mechanical performance of individual CNF was studied as well. Our findings prove that GNRs are one of the most promising reinforcements to synthesize CNFs with high strength in a facile and scalable synthesis technique.

\section{Experimental Section}

\subsection{Materials}

PAN $(M w=150,000)$ was obtained from Scientific Polymer products. Inc. Analytical pure N-N dimethyl formamide (DMF) was received from Chengdu KESHI Chemical Co. Ltd. The GNRs were prepared from CNTs with $\mathrm{Na} / \mathrm{K}$ alloy by the method as described previously[29]. The layer number of GNRs is in the range of $\sim 30-45$, the width is $\sim 100-200 \mathrm{~nm}$ and the length is $\sim 3-5 \mu \mathrm{m}$.

\subsection{Synthesis of GNRs reinforced CNFs}

Before the step of electrospinning, PAN was completely dissolved in DMF to get 6 wt.\% PAN/DMF solution, then GNRs was added to the above solution at different weight fractions from zero to $4 \mathrm{wt} . \%$ and then dispersed by tip ultrasonic vibration at 
$80 \mathrm{~W}$ for $20 \mathrm{~min}$ and continuously stirring at $250 \mathrm{rpm}$ for 1 day at $298 \mathrm{~K}$ to obtain a homogeneous solution. We provide four pictures of the solution of PAN in DMF with 4 wt.\% GNRs at different time. As shown in Figure S1, there is no precipitate out after dispersion even for three days, indicating that GNRs are homogeneously dispersed in the PAN/DMF solution. The electrospinning device was a horizontal type (Model DFS-L-01), which was provided by Beijing Tech-Nova technology co. Ltd. The obtained black solution was placed in a $5 \mathrm{~mL}$ syringe with a capillary tip in the diameter $1 \mathrm{~mm}$. During the electrospinning, the nanofibers were collected on a high speed of $700 \mathrm{rpm}$ rotating roller, which was covered with an aluminum foil. The applied voltage was $20 \mathrm{kV}$, the flow rate of solution was $1.2 \mathrm{~mL} \mathrm{~h}^{-1}$, and the spinning distance between the tip and the collector was $20 \mathrm{~cm}$. All the electrospinning were carried out at $27 \pm 2{ }^{\circ} \mathrm{C}$ and at less than $40 \%$ relative humidity.

Then the as-spun nanofibers were dried at $80{ }^{\circ} \mathrm{C}$ under vacuum for $12 \mathrm{~h}$, followed by the stabilization at $300{ }^{\circ} \mathrm{C}$ for $3 \mathrm{~h}$ in air and carbonization at $850{ }^{\circ} \mathrm{C}$ for $3 \mathrm{~h}$ in nitrogen to produce the final GNRs reinforced CNFs.

\subsection{Microstructure Characterization}

In order to analyze the effect of GNRs embedding in the microstructue, Raman spectra were examined on a micro-Raman spectroscope (LabRAM HR Evolution) using $532 \mathrm{~nm}$ incident radiation. X-ray diffraction (XRD) patterns were recorded on DMSX-2500 PC X-ray spectrometer with $\mathrm{Cu}$ K $\alpha$ radiation $(\lambda=1.5406 \AA)$ at a scanning speed of $4^{\circ} \min ^{-1}$. The specific surface area and the pore structure of the samples were 
examined by nitrogen adsorption measurements (Belsorp Mini) at $77 \mathrm{~K}$. The morphology was observed by field emission scanning electron microscope (FESEM) and high-resolution transmission electron microscope (HRTEM, LIBRA $200 \mathrm{FE}$ at $200 \mathrm{kV})$.

\subsection{Mechanical Characterization}

The as-synthesized CNFs were first loaded onto a piece of silicon wafer with 20 $\mathrm{nm} \mathrm{Au}$ on surface. The silicon wafer together with CNFs was placed under an optical microscope equipped with an objective lens $(20 \mathrm{X})$ and an eyepiece (15 X). Generally, the nanofibers with diameter of $60 \sim 80 \mathrm{~nm}$ are barely seen, while those with diameter $\sim 100 \mathrm{~nm}$ can be easily located and picked up with a fine tungsten probe (The Micromanipulator Co. Model: 7A) which is installed to a mechanical arm operated by hand. The weak van der Waals force makes it possible to attach individual CNF to the probe. To load a CNF onto the micromechanical device, the CNF should rub against the device several times, then the CNF came off and laid across the gap. The alignment of the CNFs can be tuned by slightly pushing the end of CNF. The CNFs with different diameters were placed onto the micromechanical devices with good alignment. Both ends of CNF were anchored onto the device shuttle with two drops of epoxy. In case of the epoxy flowed over the CNF, the epoxy was cured $15 \mathrm{~min}$ at ambient condition before use. The micromechanical device together with CNF was attached onto an aluminum stub using crystal bond (a commercial wax). The tensile test of an individual CNF was conducted in a SEM (FEI Quanta 400, FEI). A quantitative Agilent in SEM nanoindenter was used to actuate the device and also to 
measure the load and displacement independently. The fracture surfaces of all tested CNFs were examined after mechanical failure.

\section{Results and Discussion}

Fig. 1 displays a schematic diagram for the preparation of GNRs reinforced CNFs using an electrospun method. The GNRs were prepared through splitting CNT by alkali metal $(\mathrm{Na} / \mathrm{K})$ source[29]. The obtained GNR had integral graphitic structures, active carboanionic edges and less defects.

The GNRs and PAN were then dissolved in N, N-dimethylformamide (DMF) to form a homogeneous solution, as shown in Figure S1. The GNRs can act as nanoplatelet templates to promote the formation of graphitic carbon in vicinity of PAN matrix. Then nanofibers were electrospun on a high speed rotating roller with a $1.2 \mathrm{~mL} \mathrm{~h}^{-1}$ feed rate at $20 \mathrm{kV}$. Subsequently, the nanofibers were stabilized in air at $300{ }^{\circ} \mathrm{C}$ for $3 \mathrm{~h}$ and further carbonized in nitrogen at $850{ }^{\circ} \mathrm{C}$ for $3 \mathrm{~h}$ to finalize GNRs reinforced CNFs.

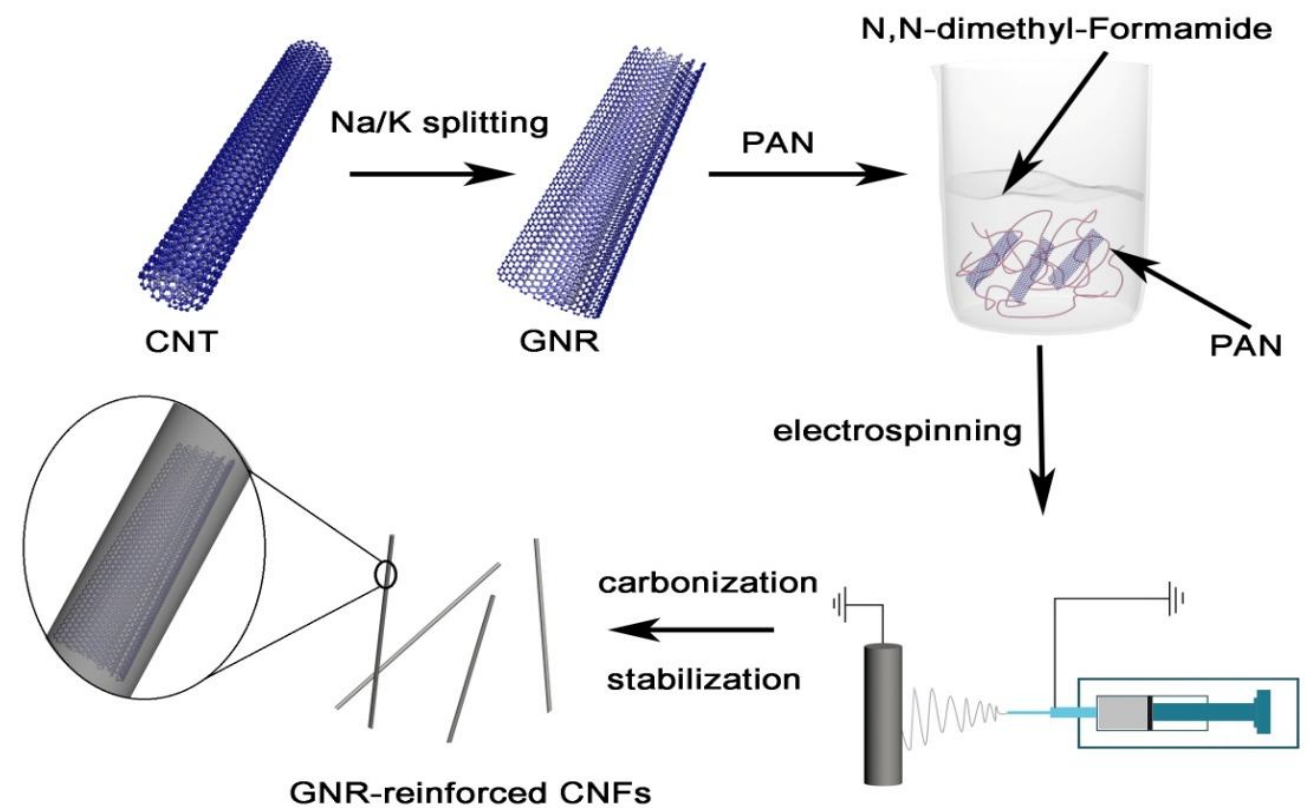


Fig. 1: Schematic diagram of preparation for GNRs-reinforced CNFs.

SEM, TEM and Raman were used to analyze the microstructure of GNRs-reinforced CNFs in detail (Fig. 2). The TEM image of original CNTs with a diameter $\sim 60 \mathrm{~nm}$ and aspect ratio $\sim 400$ for preparing GNRs can be found in the Supporting Information Figure S2a. TEM (Fig. 2a) and SEM (Figure S2b) images illustrate that CNTs are completely unzipped along the axis with opened edges to form GNRs. It can be seen that GNRs are longitudinally split, with width ranging from 72 to $215 \mathrm{~nm}$ (Fig. 2a). Fig. 2b shows the SEM image of individual GNRs-reinforced CNF with 4 wt.\% GNRs. The one-dimensional GNRs were found to be well aligned along the $\mathrm{CNF}$ axis and strongly combined with PAN matrix. More SEM images of GNRs reinforced CNFs at different GNRs weight ratio can be found in the Supplementary Information (Fig. S2c-f and Fig. S4a-d). The good alignment of GNRs and solid interface between GNRs and PAN are expected to be helpful for improving the mechanical properties of CNFs. The width of GNRs reinforced CNFs ranges from $\sim 100$ to $400 \mathrm{~nm}$. To further investigate the detailed microstructure of GNRs reinforced CNFs, the crystal phases were analyzed by X-ray diffraction (XRD). In Fig. 2c, the XRD patterns of GNRs reinforced CNFs with different GNRs weight fraction show a strong peak at $22.6^{\circ}(2 \theta)$ for all samples, which can be attributed to the typical graphite diffraction (002). The peak parameters of full width at half maximum (FWHM) of all samples were obtained by Lorentzian fitting, as shown in Table 1. The FWHM of (002) peak for GNRs reinforced GNFs is obviously reduced with the embedding of GNRs. As the GNRs weight fraction increases from zero to 4 
wt.\%, the FWHM of (002) peak decreases from $7.29^{\circ}$ to $3.17^{\circ}$, indicating a significant enhancement of graphitic carbon. The sharpened (002) peak proves that the embedding of GNRs plays an important role in promoting graphitic crystallinity for CNFs.
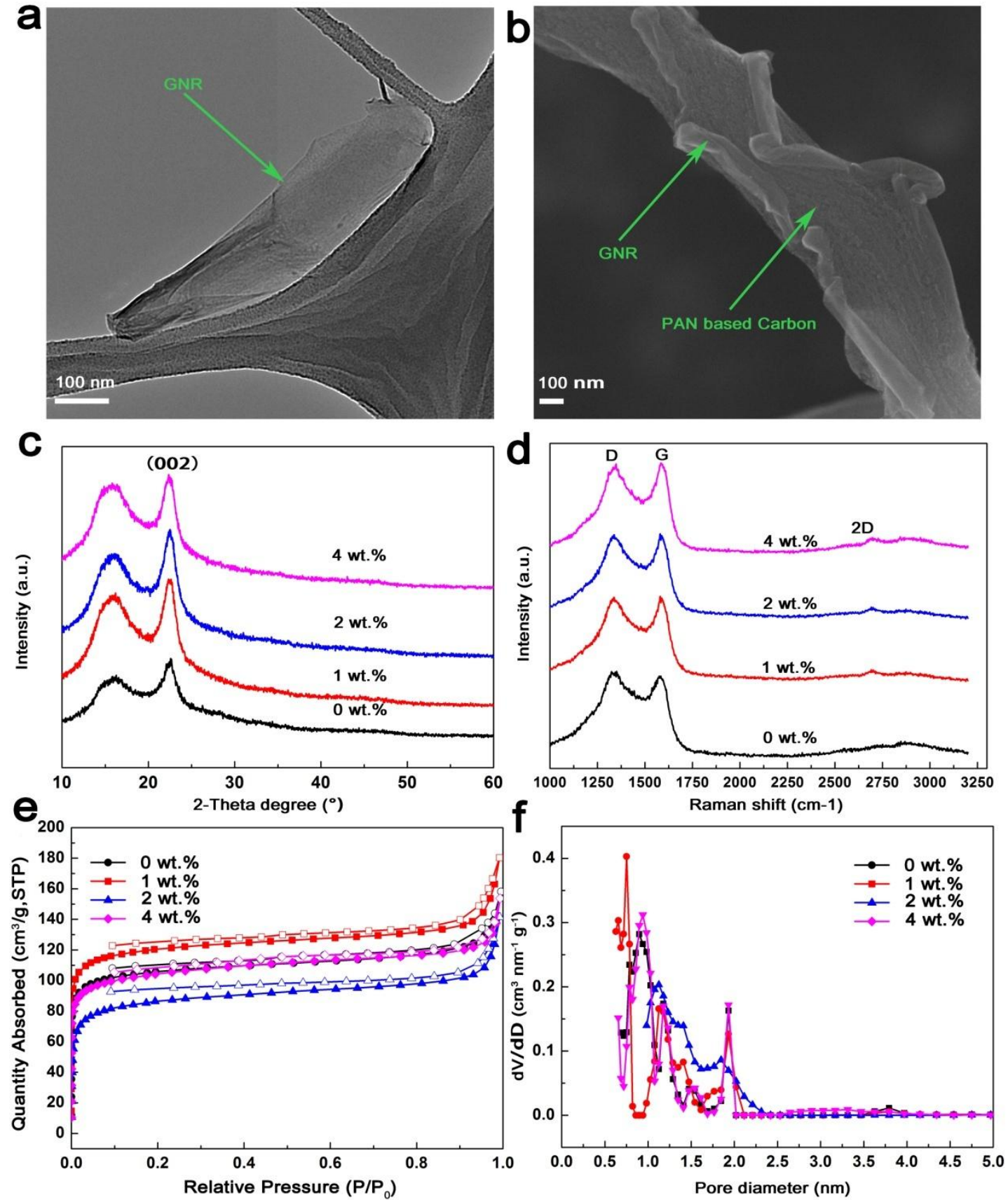

Fig. 2: (a) Transmission electron microscope (TEM) image of GNRs by Na/K splitting.

(b) Field emission scanning electron microscopy (FESEM) image of the 
GNR-reinforced CNF. (c) X-ray diffraction, (d) Raman spectra, (e) $\mathrm{N}_{2}$ adsorption and desorption curves, and (f) Pore size distribution curves of GNRs-reinforced CNFs with different GNRs weight fractions (the $y$-axis is $\mathrm{dV} / \mathrm{dD}$, or differential pore volume via pore diameter).

Table 1. Microstructure of GNR-reinforced CNFs at different GNR weight ratio

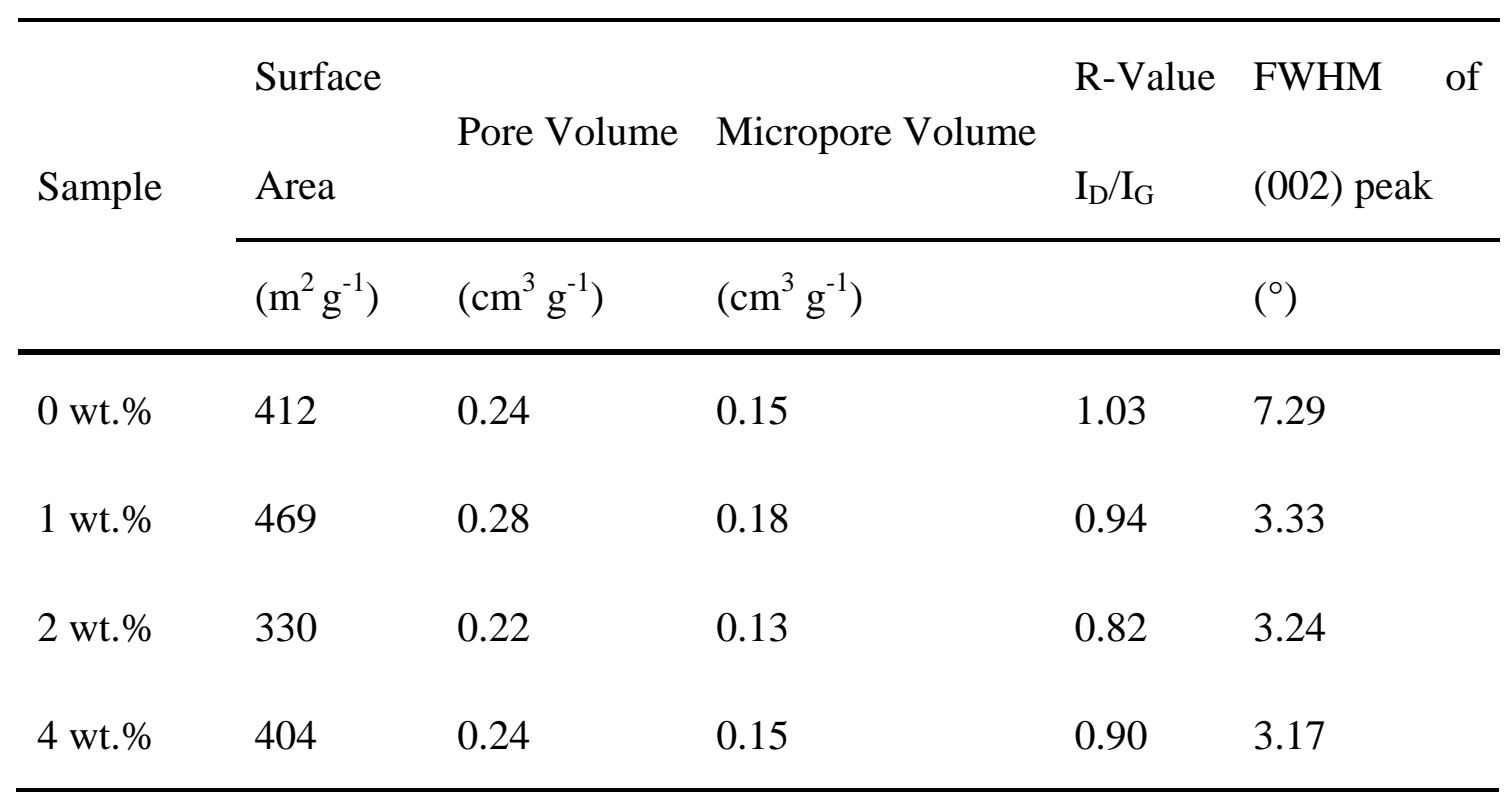

To further elucidate the graphitic structure of GNRs reinforced CNFs, Raman analysis was carried out. Fig. 2d shows Raman spectra, in which the D-band at 1350 $\mathrm{cm}^{-1}$ originates from the disordered carbon and the G-band at $1582 \mathrm{~cm}^{-1}$ associates with the ordered graphitic carbon. The relative intensity ratio of D-band to G-band, or $\mathrm{I}_{\mathrm{D}} / \mathrm{I}_{\mathrm{G}}$, which is called the "R-value", is used to quantitatively characterize the amount of structurally ordered graphite crystallites in carbonaceous materials. The $\mathrm{I}_{\mathrm{D}} / \mathrm{I}_{\mathrm{G}}$ ratio decreases from 1.03 to 0.82 when the addition of GNRs increases from 0 wt. $\%$ to 2 wt.\%, indicating that the incorporation of GNRs into PAN-based CNFs is able to effectively improve the graphitic carbon in the final products. This is in good agreement with the XRD results. In addition, Raman spectra of GONRs and GNRs 
can be found in Fig. S3. These facts reflect that GNRs not only can act as carbon nanofillers with ordered layer, but also work as nanoplatelet template to promote the growth of graphitic carbon in vicinity of PAN-based CNFs. According to previous reports[9, 20, 31], it has been demonstrated that the incorporation of CNTs into electrospun CNFs can improve the graphitic crystallization and decrease the structure defects.

To see whether there are defects in term of micro/nano pores in GNRs reinforced CNFs, we used nitrogen adsorption and desorption (Fig. 2e) to measure the specific surface area and porosity of all samples. The data of specific surface area and porosity are summarized in Table 1. The pore size distribution (PSD) curves of all samples are calculated by nonlocal density functional theory (NLDFT) and presented in Fig. $2 \mathrm{f}$. This PSD curves reveals that the four samples mainly possess micropores with the diameter less than $2 \mathrm{~nm}$. It should be noted that the CNFs with $2 \mathrm{wt} . \%$ GNRs have the smallest differential pore volume via pore diameter and the smallest total pore volume, indicating that it has less structure defects in the bulk than the other three samples. The decrease of total pore volume, which is probably due to strong interaction between GNRs and PAN matrix, the rearrangement of graphitic lamellar as well, leads to the increase of density and the reduction of defect sites. According to Weibull weakest link model $[32,33]$, fracture begins with the largest stress concentration. The reduction of pore volume can improve the mechanical performance because the amount of defect sites decrease.

Fig. 3 shows the in situ tensile test of an individual CNF reinforced by 2 wt. $\%$ 
GNRs. Both ends of the CNF are anchored by epoxy onto a micromechanical device shown in Fig. 3a. The diameter of the CNF is $161.0 \mathrm{~nm}$. The associated inset in Fig. 3a illustrates the SEM image of the micromechanical device with a quantitative indenter tip engaging on the top shuttle. Load on the specimens and the corresponding displacement can be derived from the indenter load and displacement data using a simple response subtraction method and image correlation technique[34-36]. Fig. 3b-c show low- and high-magnification SEM images of the fractured surface of the CNF after mechanical failure. Interestingly, a rough surface is found, which is desired due to that the fracture energy can be consumed during crack growth. Fig. $3 \mathrm{~d}$ is the linear stress-strain curve of the CNF. The corresponding video can be found in the Supporting Information Video S1. The stress is calculated using equation (1):

$$
\sigma=F /\left[\pi \cdot(D / 2)^{2}\right]
$$

Where $F$ is the measured force, $D$ is the diameter of the suspended CNF. The strain is calculated by equation (2):

$$
\varepsilon=\delta / L
$$

Where $\delta$ is the elongation and $L$ is the initial length of the suspended CNF. The elastic modulus and fracture strength of the CNF in Fig. $3 \mathrm{a}$ are subsequently calculated to be 70.04 $\mathrm{GPa}$ and $3.52 \mathrm{GPa}$, respectively. In order to further confirm the measured values, we fractured six more CNFs with different diameters. The average elastic modulus and fracture strength are 55.12 $\pm 14.92 \mathrm{GPa}$ and $3.35 \pm 0.75 \mathrm{GPa}$, respectively. And the reinforcement effect of GNRs in tensile strength and elastic modulus are shown in Fig. 3e-f. Table 2 lists the measured samples diameter, fracture strength, and 
elastic modulus of CNFs. The size and distribution of GNRs embedded in CNFs are not controlled uniformly at each fraction, resulting in scattered mechanical performances, as shown in Fig.3e-f. And CNFs with more structural defects tend to exhibit smaller Weibull modulus, which suggests that the defects amount increase the randomness of the mechanical performance [1-3]. But there still clearly appears a trend that the embedding of GNRs was effective to improve the tensile strength and elastic modulus of CNFs with a similar diameter when the addition increases from 0 wt. $\%$ to 2 wt. $\%$. 

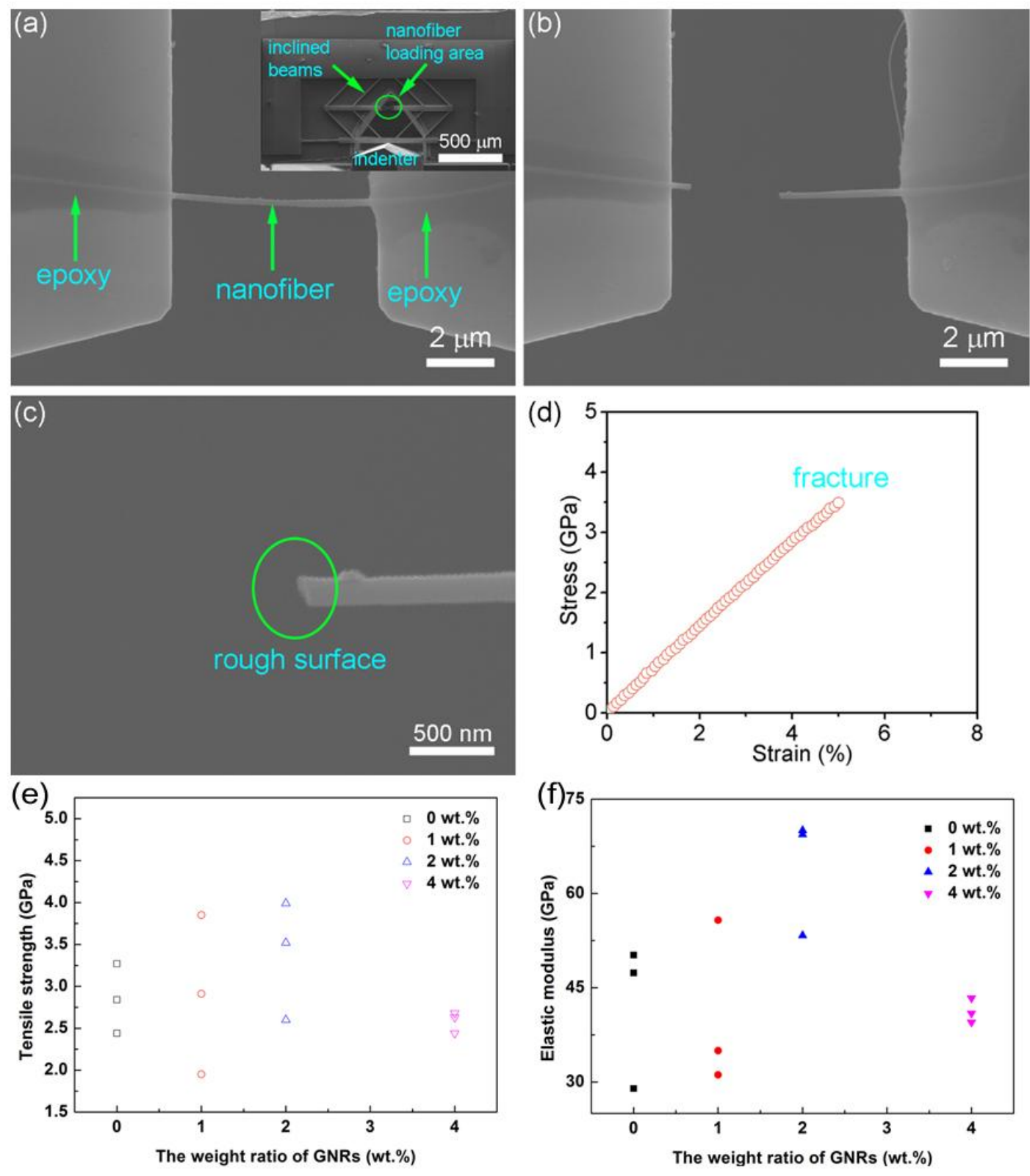

Fig. 3: In situ tensile test of CNF with GNRs embedding. (a) SEM image of a CNF with 2 wt.\% GNRs in a diameter of $161.0 \mathrm{~nm}$ before tensile test with both ends anchored by epoxy onto a micromechanical device. The inset in (a) SEM image of the micromechanical device with an indenter tip engaging on the top shuttle. (b) SEM image of the CNF after tensile test. (c) High-magnification SEM image of fracture surface of the CNF. (d) Stress-strain curve of the CNF. (e) Tensile strength of CNFs at different weight ratio of GNRs. (f) Elastic modulus of CNFs at different weight ratio 
of GNRs.

Table 2. Diameter, tensile strength, and elastic modulus of GNR-reinforced CNFs

\begin{tabular}{|c|c|c|c|}
\hline \multirow{2}{*}{ Sample } & \multirow{2}{*}{$\begin{array}{l}\text { Sample Diameter } \\
(\mathrm{nm})\end{array}$} & \multirow{2}{*}{$\begin{array}{l}\text { Tensile Strength } \\
(\mathrm{GPa})\end{array}$} & \multirow{2}{*}{$\begin{array}{l}\text { Elastic Modulus } \\
(\mathrm{GPa})\end{array}$} \\
\hline & & & \\
\hline & 121.1 & 2.84 & 47.38 \\
\hline \multirow[t]{3}{*}{0 wt. $\%$} & 128.0 & 3.27 & 50.21 \\
\hline & 160.6 & 2.44 & 28.97 \\
\hline & 164.9 & 1.95 & 31.15 \\
\hline \multirow[t]{3}{*}{1 wt.\% } & 170.2 & 3.85 & 55.72 \\
\hline & 175.8 & 2.91 & 34.98 \\
\hline & 161.0 & 3.52 & 70.04 \\
\hline \multirow[t]{3}{*}{2 wt. $\%$} & 170.2 & 2.60 & 53.31 \\
\hline & 179.0 & 3.99 & 69.40 \\
\hline & 189.7 & 2.44 & 39.49 \\
\hline \multirow[t]{2}{*}{4 wt.\% } & 202.2 & 2.63 & 43.36 \\
\hline & 218.8 & 2.68 & 40.92 \\
\hline
\end{tabular}

To learn the effect of GNRs on the mechanical performance of CNFs, the pristine PAN based CNFs, CNFs reinforced by $1 \mathrm{wt} . \%$ and $4 \mathrm{wt} . \%$ GNRs were fractured as well. For each group, at least 10 specimens were tested. However, all CNFs were picked up and loaded onto the micromechanical device under an optical microscope. 
The diameter of CNFs ranges from $110 \mathrm{~nm}$ to $220 \mathrm{~nm}$, which is hard to be exactly measured before test in SEM. The diameter, tensile strength and elastic modulus of selected pristine CNFs and GNRs reinforced CNFs are listed in Table 2. The scattered tensile strength and elastic modulus might result from the defects in fiber, the non-uniform distribution of GNRs, irregular conformation of GNRs and variety in the different GNR-PAN interface condition. However, the elastic modulus strongly depends on the diameter, even for those CNFs with same GNR weight ratio, which is consistent with previous reports $[1,3,16,37]$. The diameter of CNFs tends to enlarge with the increase of GNRs embedding, as shown in Fig.S5. Fibers with smaller diameters yield higher mechanical properties owing to minimization of structure defects. The tensile strength of pristine PAN-based CNF is $2.85 \pm 0.41 \mathrm{GPa}$, which is in accordance with the previous work of Weibull modulus[15]. With GNRs increasing from zero to $2 \mathrm{wt} . \%$, the tensile strength and elastic modulus of CNFs are obviously improved, especially those fibers reinforced by 2 wt.\% GNRs. For example, the tensile strength and modulus of pristine PAN-based CNF with diameter of $161 \mathrm{~nm}$ is 2.44 and $28.97 \mathrm{GPa}$, respectively, while these values significantly increase to 3.52 and $70.04 \mathrm{GPa}$ for the CNFs reinforced by 2 wt.\% GNRs, reflecting the strong reinforcement of GNRs. According to the microstructure analysis, CNFs with 2 wt.\% GNRs embedding possess a higher graphitic crystallinity, smaller pore volume, and less structural defects, which can result in higher tensile strength and modulus. In addition to the desired microstructure, the combination of GNRs with PAN matrix along the CNF axis and good interfacial bonding between reinforcement and matrix 
can also enhance the mechanical performance. Interestingly, for the CNFs reinforced by 4 wt.\% GNRs, the diameter of the fibers is the largest among all four kinds of samples and the mechanical properties are even lower than those of CNFs with 2 wt.\% GNR inside. The larger sample size suggests that the probability of micropores and defects existence increases, which would further weaken the mechanical performance of CNFs. According to the stress calculation, tensile strength is found to be inversely proportional to diameter square.

Fracture surfaces of all CNFs reinforced by GNRs were post observed by high-resolution SEM (Figure 4). Fig. 4a-d shows SEM images of fracture surfaces of CNFs reinforced by 0 wt. $\%, 1$ wt. $\%, 2$ wt. $\%$, and 4 wt.\% GNRs after mechanical failure. In Fig. 4a, it can be clearly seen that the fracture surface of the pristine CNF is smooth, indicating brittle fracture behavior. A single neck pointed out by an arrow was observed under tensile deformation, which was consistent with previous work [38]. The necking may appear in the sites of a low molecular entanglement density or disordered carbon in the outer layer, resulting from the electrospun preparation of nonreinforced CNFs. It should be noted that the nanofibers were highly stretched and solidified on a high speed rotating wheel in a short time during electrospinning. In contrast, the ruptured surfaces of GNRs reinforced CNFs are much rougher. The SEM images in Fig. 4b-d show a protruding GNR on the ends of CNFs. Specifically, Fig. 4e-f shows a sword-in-sheath fracture mode in $4 \mathrm{wt} \%$ GNRs reinforced CNFs, confirming that the GNRs were well embedded in the CNF along the axis and acted as strong reinforcements for CNFs. Brown et al.[2,4], observed a cup-and-cone style of 
fracture surface on amorphous carbon fibers with CNTs embedding, as a result from the plastic deformation on the outer shell and the protruding of inner layers. In our work, the outer shell, made from the carbonization of PAN polymer, was initially plastic deformed and eventually fractured at the defect sites. The inner layer where the GNRs were embedded with ordered carbon was much stronger than the outer layer. Therefore, the inner layer was subsequently slid and pulled out, resulting in the sword-in-sheath style. Fig. $4 \mathrm{~g}$ shows the schematic illustration of sword-in-sheath mode of the fracture surface. 

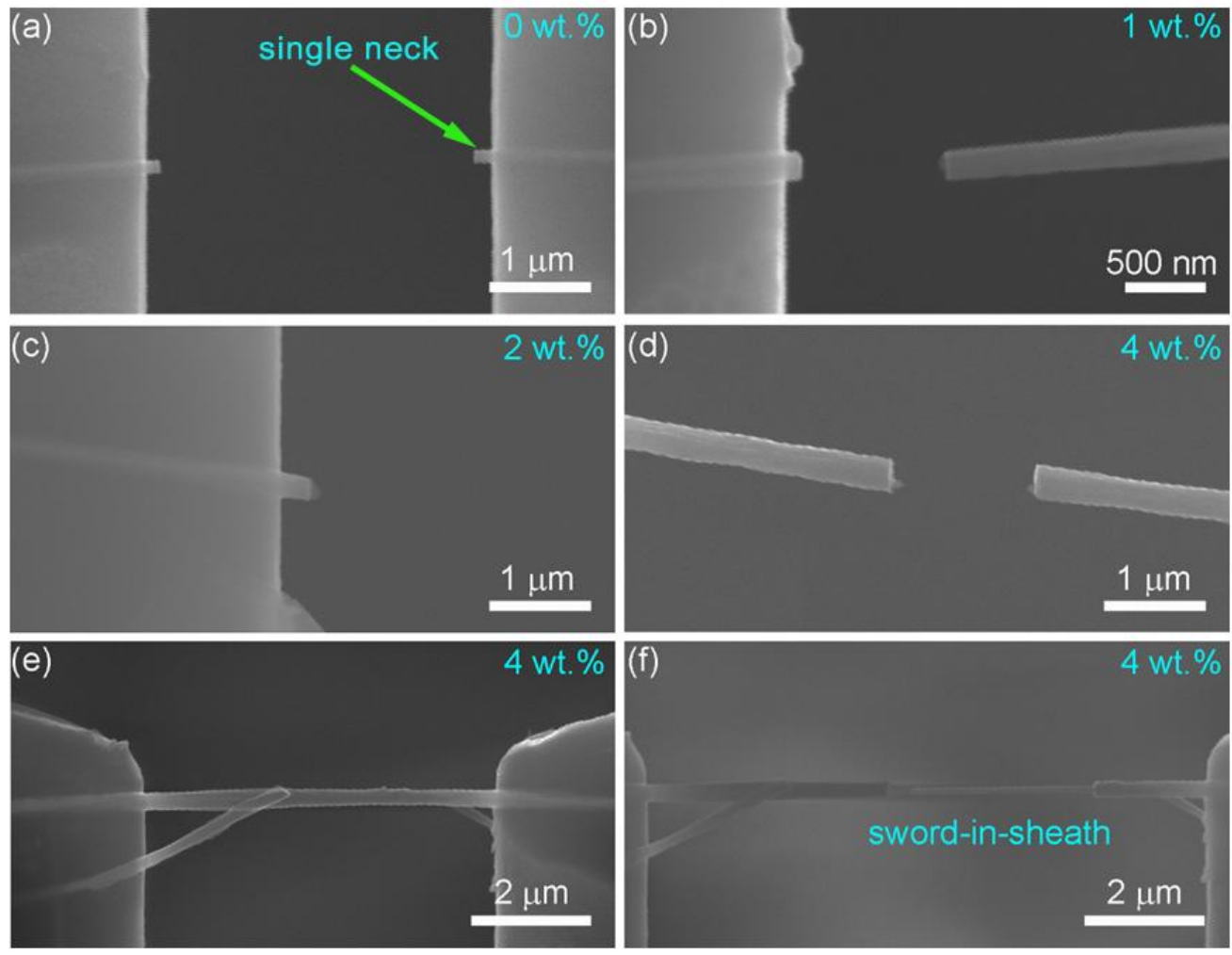

(g)

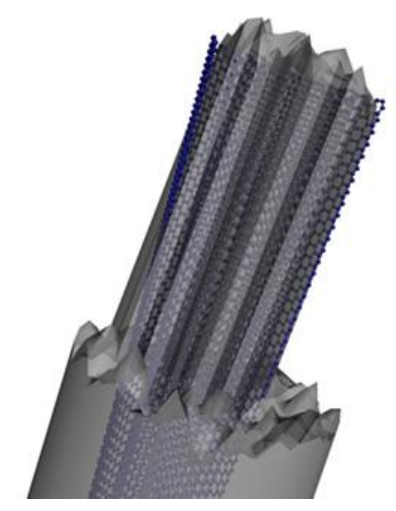

Fig. 4: Fracture surfaces of CNFs with different weight fraction of GNRs inside (a)

SEM image of a fractured pristine CNF. (b-d) SEM images of fractured nanofibers with 1 wt.\%, 2 wt.\%, and 4 wt.\% GNRs adding, respectively. (e) SEM image of a CNF with 4 wt.\% GNRs embedding before tensile testing. (f) The CNF in (e) fractured following the sword-in-sheath style. (g) Schematic fracture illustration of individual GNR-reinforced CNF after tensile test. 


\section{Conclusion}

GNRs reinforced PAN-based CNFs are synthesized by electrospinning, stabilized in air at $300{ }^{\circ} \mathrm{C}$ for $3 \mathrm{~h}$ and carbonized in nitrogen at $850{ }^{\circ} \mathrm{C}$ for $3 \mathrm{~h}$. SEM images, X-ray diffraction, and Raman spectroscopy show that GNRs were entirely embedded along the fiber axis and can effectively enhance the formation of graphitic carbon in CNFs. In situ tensile tests are carried out on individual CNFs using a micromechanical device inside the SEM. When the embedding content of GNRs increases from 0 to $2 \mathrm{wt} \%$, tensile strength and elastic modulus of CNFs can be significantly improved due to the increase of graphitic carbon and decrease of porosity. The rough fracture surfaces are observed in GNRs reinforced CNFs, which can consume more fracture energy and subsequently pull off the catastrophic failure. This study demonstrates that the GNRs reinforced PAN-based CNFs alone should be one of the most promising structure materials and as an exceptional reinforcement also can find more application in composites.

\section{Acknowledgements}

This work was funded by the Natural Science Foundation Project of CQ CSTC CHINA (No.2015jcyjA50020) and China Scholarship Council (No. 201406055002). Y.Y and J.L would like to acknowledge the financial support by the US Air Force Office of Scientific Research (Grant FA9550-13-1-0084) and the US Department of Energy, Office of Basic Energy Sciences (Contract DE-FG02-13ER46967). X.L. Li and Y.C. Yang contributed equally to this work.

\section{Appendix A. Supplementary data}


Supplementary data related to this article can be found at \#\#\#\#\#\#\# or from the author.

\section{References}

[1] Beese, A.M., D. Papkov, S.Y. Li, Y. Dzenis, H.D. Espinosa, In situ transmission electron microscope tensile testing reveals structure-property relationships in carbon nanofibers. Carbon, 2013. 60: p. 246-253.

[2] Zhang, J.N., P. Loya, C. Peng, V. Khabashesku, J. Lou, Quantitative In Situ Mechanical Characterization of the Effects of Chemical Functionalization on Individual Carbon Nanofibers. Adv. Funct. Mater. 2012. 22(19): p. 4070-4077.

[3] Yang, Y., W. Chen, E. Hacopian, P. Dong, A. Sun, L. Ci, et al., Unveil the Size-Dependent Mechanical Behaviors of Individual CNT/SiC Composite Nanofibers by In Situ Tensile Tests in SEM. Small, 2016. 12(33): p. 4486-91.

[4] Brown, J.J., J.W. Suk, G. Singh, A.I. Baca, D.A. Dikin, R.S. Ruoff, et al., Microsystem for nanofiber electromechanical measurements. Sens. Actuators A, 2009. 155(1): p. 1-7.

[5] Song, H.Y. W.Z. Shen, Carbon Nanofibers: Synthesis and Applications. J. Nanosci. Nanotechno. 2014. 14(2): p. 1799-1810.

[6] Gao, J., M. Hu, Y. Dong, and R.K. Li, Graphite-nanoplatelet-decorated polymer nanofiber with improved thermal, electrical, and mechanical properties. ACS Appl. Mater. Interfaces, 2013. 5(16): p. 7758-64.

[7] Johannsen, I., K. Jaksik, N. Wirch, P. Pötschke, B. Fiedler, K. Schulte, Electrical conductivity of melt-spun thermoplastic poly(hydroxy ether of 
bisphenol A) fibres containing multi-wall carbon nanotubes. Polymer, 2016. 97: p. 80-94.

[8] Chien, A.-T., H.C. Liu, B.A. Newcomb, C. Xiang, J.M. Tour, S. Kumar, Polyacrylonitrile Fibers Containing Graphene Oxide Nanoribbons. ACS Appl. Mater. Interfaces, 2015. 7(9): p. 5281-5288.

[9] Chae, H.G., M.L. Minus, A. Rasheed, S. Kumar, Stabilization and carbonization of gel spun polyacrylonitrile/single wall carbon nanotube composite fibers. Polymer, 2007. 48(13): p. 3781-3789.

[10] Matsumoto, H., S. Imaizumi, Y. Konosu, M. Ashizawa, M. Minagawa, A. Tanioka, et al., Electrospun composite nanofiber yarns containing oriented graphene nanoribbons. ACS Appl. Mater. Interfaces, 2013. 5(13): p. 6225-31.

[11] Wu, S., F. Zhang, Y. Yu, P. Li, X. Yang, J. Lu, et al., Preparation of PAN-based carbon nanofibers by hot-stretching. Compos. Interface, 2008. 15(7-9): p. 671-677.

[12] Song, Z., X. Hou, L. Zhang, S. Wu, Enhancing Crystallinity and Orientation by Hot-Stretching to Improve the Mechanical Properties of Electrospun Partially Aligned Polyacrylonitrile (PAN) Nanocomposites. Materials, 2011. 4(12): p. 621-632.

[13] Liu, Y.D. S. Kumar, Recent Progress in Fabrication, Structure, and Properties of Carbon Fibers. Polym. Rev, 2012. 52(3-4): p. 234-258.

[14] Rahaman, M.S.A., A.F. Ismail, A. Mustafa, A review of heat treatment on polyacrylonitrile fiber. Polym. Degrad. Stabil, 2007. 92(8): p. 1421-1432. 
[15] Arshad, S.N., M. Naraghi, I. Chasiotis, Strong carbon nanofibers from electrospun polyacrylonitrile. Carbon, 2011. 49(5): p. 1710-1719.

[16] Wang, Z., P. Poncharal, W. De Heer, Measuring physical and mechanical properties of individual carbon nanotubes by in situ TEM. J. Phys. Che. Solids, 2000. 61(7): p. 1025-1030.

[17] Richard-Lacroix, M., C. Pellerin, Molecular Orientation in Electrospun Fibers: From Mats to Single Fibers. Macromolecules, 2013. 46(24): p. 9473-9493.

[18] Inaba, K., K. Saida, P. Ghosh, K. Matsubara, M. Subramanian, A. Hayashi, et al., Determination of Young's modulus of carbon nanofiber probes fabricated by the argon ion bombardment of carbon coated silicon cantilever. Carbon, 2011. 49(13): p. 4191-4196.

[19] Hou, X., X. Yang, L. Zhang, E. Waclawik, S. Wu, Stretching-induced crystallinity and orientation to improve the mechanical properties of electrospun PAN nanocomposites. Mate. Design, 2010. 31(4): p. 1726-1730.

[20] Cai, J., S. Chawla, M. Naraghi, Microstructural evolution and mechanics of hot-drawn CNT-reinforced polymeric nanofibers. Carbon, 2016.

[21] Sahin, K., N.A. Fasanella, I. Chasiotis, K.M. Lyons, B.A. Newcomb, M.G. Kamath, et al., High strength micron size carbon fibers from polyacrylonitrile-carbon nanotube precursors. Carbon, 2014. 77: p. 442-453.

[22] Li, Q., J.S. Church, M. Naebe, B.L. Fox, Interfacial characterization and reinforcing mechanism of novel carbon nanotube - Carbon fibre hybrid composites. Carbon, 2016. 109: p. 74-86. 
[23] Chae, H.G., Y.H. Choi, M.L. Minus, S. Kumar, Carbon nanotube reinforced small diameter polyacrylonitrile based carbon fiber. Compos. Sci. Technol, 2009. 69(3-4): p. 406-413.

[24] Ge, J.J., H. Hou, Q. Li, M.J. Graham, A. Greiner, D.H. Reneker, et al., Assembly of well-aligned multiwalled carbon nanotubes in confined polyacrylonitrile environments: electrospun composite nanofiber sheets. J. Am. Chem.1 Soc., 2004. 126(48): p. 15754-15761.

[25] Ji, J., G. Sui, Y. Yu, Y. Liu, Y. Lin, Z. Du, et al., Significant improvement of mechanical properties observed in highly aligned carbon-nanotube-reinforced nanofibers. J. Phy. Chem. C, 2009. 113(12): p. 4779-4785.

[26] Laird, E.D. C.Y. Li, Structure and Morphology Control in Crystalline Polymer-Carbon Nanotube Nanocomposites. Macromolecules, 2013. 46(8): p. 2877-2891.

[27] Xiang, C., N. Behabtu, Y. Liu, H.G. Chae, C.C. Young, B. Genorio, et al., Graphene nanoribbons as an advanced precursor for making carbon fiber. ACS nano, 2013. 7(2): p. 1628-1637.

[28] Lu, W., G. Ruan, B. Genorio, Y. Zhu, B. Novosel, Z. Peng, et al., Functionalized graphene nanoribbons via anionic polymerization initiated by alkali metal-intercalated carbon nanotubes. ACS nano, 2013. 7(3): p. 2669-2675.

[29] Genorio, B., W. Lu, A.M. Dimiev, Y. Zhu, A.-R.O. Raji, B. Novosel, et al., In situ intercalation replacement and selective functionalization of graphene 
nanoribbon stacks. ACS nano, 2012. 6(5): p. 4231-4240.

[30] Shang, S.M., L. Gan, C.W.M. Yuen, S.X. Jiang, N.M. Luo, The synthesis of graphene nanoribbon and its reinforcing effect on poly (vinyl alcohol). Compos. Part a-Appl. S, 2015. 68: p. 149-154.

[31] Papkov, D., A.M. Beese, A. Goponenko, Y. Zou, M. Naraghi, H.D. Espinosa, et al., Extraordinary Improvement of the Graphitic Structure of Continuous Carbon Nanofibers Templated with Double Wall Carbon Nanotubes. Acs Nano, 2013. 7(1): p. 126-142.

[32] Lachman, N., B.J. Carey, D.P. Hashim, P.M. Ajayan, H.D. Wagner, Application of continuously-monitored single fiber fragmentation tests to carbon nanotube/carbon microfiber hybrid composites. Compos. Sci. Technol, 2012. 72(14): p. 1711-1717.

[33] Yavin, B., H. Gallis, J. Scherf, A. Eitan, H. Wagner, Continuous monitoring of the fragmentation phenomenon in single fiber composite materials. Polym. Compos, 1991. 12(6): p. 436-446.

[34] Lu, Y., Y. Ganesan, J. Lou, A multi-step method for in situ mechanical characterization of 1-D nanostructures using a novel micromechanical device. Exp. Mech, 2010. 50(1): p. 47-54.

[35] Ganesan, Y., Y. Lu, C. Peng, H. Lu, R. Ballarini, J. Lou, Development and application of a novel microfabricated device for the in situ tensile testing of 1-D nanomaterials. J. MEMS, 2010. 19(3): p. 675-682.

[36] Ganesan, Y., C. Peng, Y. Lu, P.E. Loya, P. Moloney, E. Barrera, et al., 
Interface toughness of carbon nanotube reinforced epoxy composites. ACS Appl. Mater. Interfaces, 2011. 3(2): p. 129-134.

[37] Liu, L.Q., D. Tasis, M. Prato, H.D. Wagner, Tensile mechanics of electrospun multiwalled nanotube/poly (methyl methacrylate) nanofibers. Adv. Mater, 2007. 19(9): p. 1228-1233.

[38] Callister, W.D., Fundamentals of materials science and engineering: an interactive e-text. $5^{\text {th }}$ ed, New York 2001.p.176 


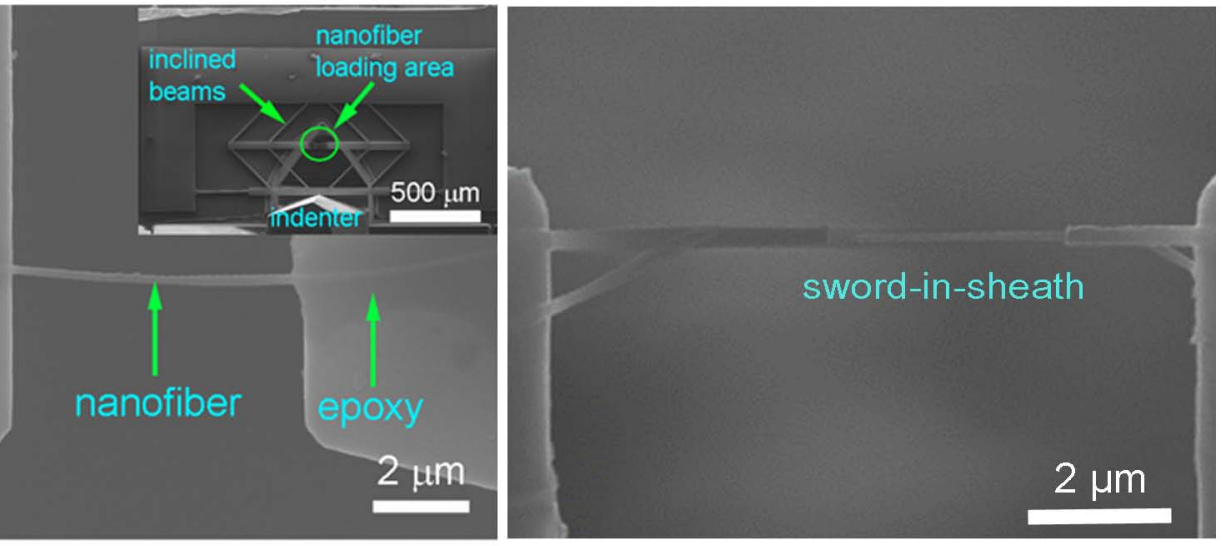

